\title{
Propensity for Risk Taking Across the Life Span and Around the Globe
} (e)

\author{
Rui Mata' ${ }^{1}$, Anika K. Josef ${ }^{2}$, and Ralph Hertwig ${ }^{2}$ \\ ${ }^{1}$ Department of Psychology, University of Basel, and ${ }^{2}$ Center for Adaptive Rationality, Max Planck Institute \\ for Human Development, Berlin, Germany
}

\begin{abstract}
Past empirical work suggests that aging is associated with decreases in risk taking. But are such effects universal? Lifehistory theory suggests that the link between age and risk taking is a function of specific reproductive strategies that can be more or less risky depending on the ecology. We assessed variation in the age-risk curve using World Values Survey data from 77 countries $(N=147,118)$. The results suggest that propensity for risk taking tends to decline across the life span in the vast majority of countries. In addition, there is systematic variation among countries: Countries in which hardship (e.g., high infant mortality) is higher are characterized by higher levels of risk taking and flatter age-risk curves. These findings suggest that hardship may function as a cue to guide life-history strategies. Age-risk relations thus cannot be understood without reference to the demands and affordances of the environment.
\end{abstract}

\section{Keywords}

risk taking, adult development, gender differences, cross-cultural differences, open materials

Received 3/11/15; Revision accepted 10/27/15

How does propensity for risk taking change across the life span and around the world? Several lines of evidence suggest that propensity for risky behavior increases in adolescence, peaks in young adulthood, and declines with aging (Dohmen et al., 2011; Mandal \& Roe, 2014; Quetelet, 1842/2013). One paradigmatic example of this progression is the link between age and criminal behavior-one possible manifestation of risk taking that has been well documented since the 19th century (Quetelet, 1842/2013). The relation between age and crime has been replicated in different cohorts and cultures, albeit with significant variation (Steinberg, 2013; Ulmer \& Steffensmeier, 2014), but to what extent do cultures vary systematically in age-risk progression?

One influential conception of risk taking is that it serves a functional role (i.e., an adaptation) that may be biologically determined (Mishra, 2014; Sih \& Del Giudice, 2012; Wilson \& Daly, 1985). In line with this view, propensity for risk taking and associated constructs, such as impulsivity and sensation seeking, have been conceptualized as traits with strong biological underpinnings (Steinberg, 2008; Zuckerman, 2007) that show moderate to high heritability (Anokhin, Golosheykin, Grant, \& Heath, 2012; Benjamin et al., 2012; Bezdjian, Baker, \& Tuvblad, 2011) and reliable gender differences (Cross, Copping, \& Campbell, 2011; Cross, Cyrenne, \& Brown, 2013).

The view that risk taking serves a functional role is best discussed in the context of life-history theory, a framework that addresses how organisms allocate time and energy to tasks and traits so as to maximize their fitness. This framework focuses particularly on how evolutionary forces shape the timing of life events involved in development, growth, and reproduction as a result of ecological characteristics (Kaplan \& Gangestad, 2005). According to life-history theory, even universal adaptations "may be limited by sex, life history stage, or circumstance" (Tooby \& Cosmides, 1990, p. 393). In other words, life-history strategies, such as reproductive strategies, can be expected to change as a function of ecological circumstances.

\footnotetext{
Corresponding Author:

Rui Mata, Center for Cognitive and Decision Sciences, Department of Psychology, University of Basel, Missionsstrasse 64A, 4055 Basel, Switzerland

E-mail: rui.mata@unibas.ch
} 
Current views suggest that one can frame human reproductive strategies along a continuum (Ellis et al., 2012): Toward one end, individuals may adopt a slower life-history strategy that focuses on avoiding risks and producing a few high-quality offspring that are likely to survive and reproduce; toward the other end, individuals may adopt faster life strategies that consist of taking risks in the service of promoting mating opportunities, early reproduction, and a greater number of offspring with more variable outcomes. These risky strategies may be particularly adaptive in harsh environments, in which morbidity and mortality are high and individuals have to compete fiercely for resources. In contrast, in rich, predictable environments, a slower reproductive strategy could be more appropriate. In other words, the rationale is that harsh, unpredictable environments may lead individuals to gamble on shorter life spans and earlier reproduction, given that fitness is likely enhanced by breeding early and abundantly rather than wasting resources on promoting one's own (unlikely) survival in such conditions (Ellis et al., 2012; Frankenhuis \& de Weerth, 2013). There is indeed empirical evidence of the dependency between reproductive strategies and the harshness or unpredictability of local environments (Belsky, Schlomer, \& Ellis, 2012; Simpson, Griskevicius, Kuo, Sung, \& Collins, 2012; Wilson \& Daly, 1997). Life-history theory also suggests that risky behaviors can be expected to be more prevalent among males, who are more likely than females to face reproductive competition (Ellis et al., 2012).

In sum, local conditions, such as the availability of resources and associated competition, are likely to affect individuals' propensity for risk taking. Ultimately, such factors may play a role in determining the shape of the age-risk relation, and resource scarcity and hardship may lead to longer periods of risk taking across the adult life span. In the present study, we tested whether local conditions of hardship could be used to predict cross-cultural variation in risk taking across the life span.

\section{The Present Study}

There has been considerable interest in accounting for similarities and differences in risk taking between cultures and countries (Becker, Dohmen, Enke, \& Falk, 2014; Hsee \& Weber, 1999; Rieger, Wang, \& Hens, 2015; Vieider et al., 2015). However, this past work has not considered the extent to which the propensity to take risks is associated with age across cultures. We aimed to contribute to this effort by investigating the following research questions: Is a universal progression of risk propensity associated with age, such that risk propensity declines from adulthood to old age? Do local characteristics (e.g., exposure to hardship), as well as age and gender differences, account for potential differences in risk taking across cultures?
To answer these questions, we analyzed data from the World Values Survey (http://www.worldvaluessurvey .org), which aims to explore people's values and beliefs around the globe. It consists of a series of nationally representative surveys of various countries in which similar questionnaires are used, mostly in face-to-face interviews. We analyzed data collected in the last two independent waves of the survey (World Values Survey Association, 2008, 2014), which included one item we take to measure propensity for risk taking. Data for this item were available for 77 countries (see Fig. 1). In particular, participants were asked to report their similarity to a hypothetical individual: "Adventure and taking risks are important to this person; to have an exciting life" (for details, see Method). This item stems from Schwartz's (2012) Value Survey, which was designed to tap into a set of 10 independent universal values. According to Schwartz, this item captures individuals' need for variety and stimulation to maintain an optimal level of activation, and relates to feelings of excitement, variety seeking, and daringness. We take this item to measure the closely linked constructs of propensity for risk taking and sensation seeking that are empirically and theoretically related. For example, sensation seeking has been characterized as "a trait defined by the seeking of varied, novel, complex, and intense sensations and experiences, and the willingness to take physical, social, legal, and financial risks for the sake of such experience" (Zuckerman, 2007, p. 27).

Our analytic strategy proceeded in two steps. First, we analyzed the link between age and propensity for risk taking across the 77 countries. Second, we examined the extent to which a measure of exposure to hardship (i.e., a composite index capturing economic and social hardship through measures of gross domestic product per capita, homicide rate, and income inequality, among others) could account for cross-country variation in the pattern of the propensity for risk taking across the life span. The rationale for the latter analysis was to test the expectation that countries in which individuals are most exposed to hardship are likely to show higher levels of risk taking and longer periods of risk taking across the adult life span.

\section{Method}

\section{Participants and procedure}

We used data from the last two independent waves, Waves 5 and 6, of the World Values Survey (World Values Survey Association, 2008, 2014). We based our analysis on a balanced sample that consisted of respondents with valid answers on the risk item as well as all covariates of interest. Our final sample comprised 147,118 individuals 


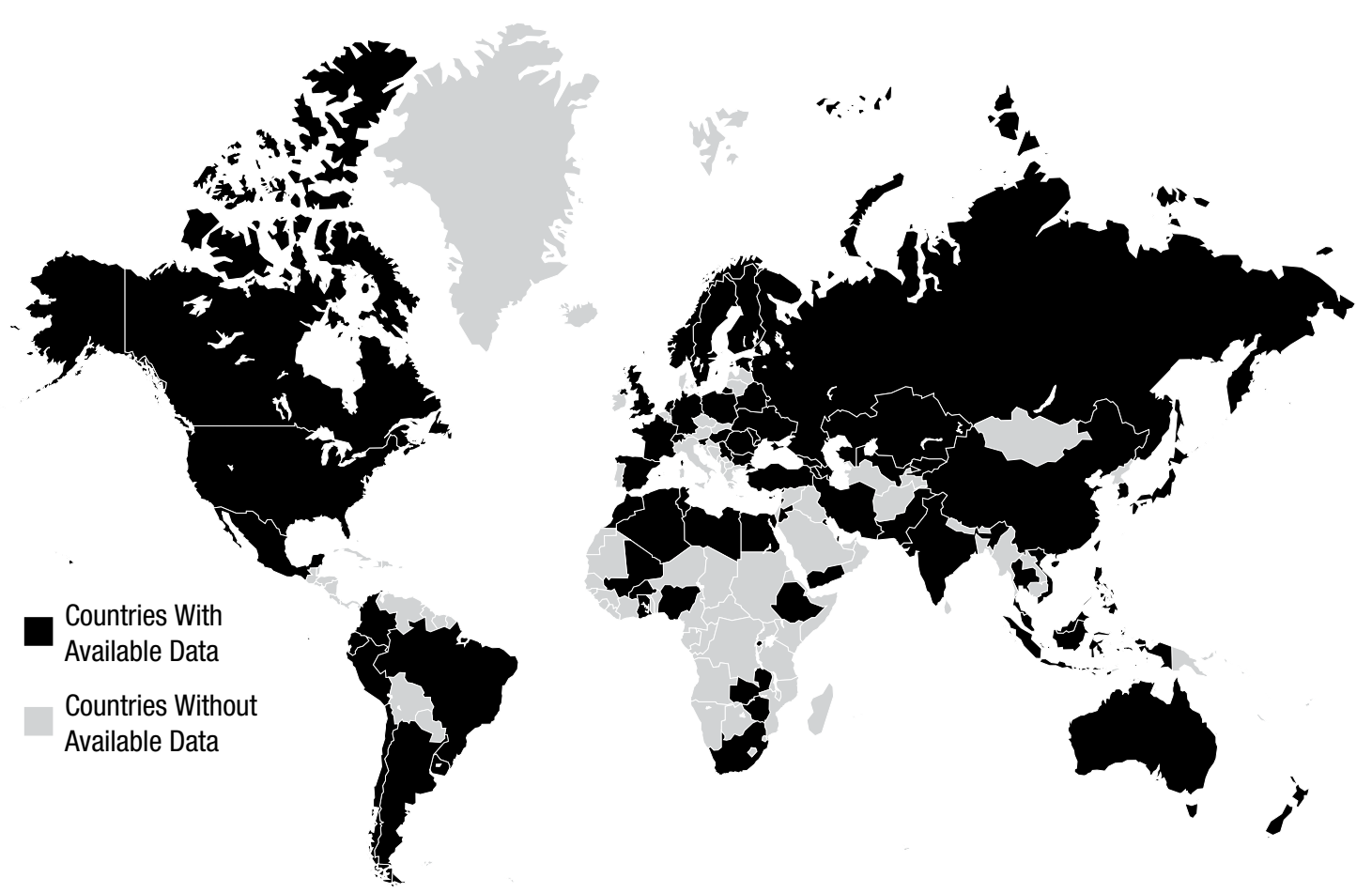

Fig. 1. World map showing countries for which data on the measure of propensity for risk taking were available from the World Values Survey.

(76,617 females, 52\%; age range $=15-99$ years $)$. The countries included in the analysis cover the full range of global variation, from very poor to very rich countries, in all of the world's major cultural zones.

\section{Measures}

Propensity for risk taking and demographic covariates. Each respondent in the World Values Survey (World Values Survey Association, 2008, 2014) heard the following information:

Now I will briefly describe some people. Using this card, would you please indicate for each description whether that person is very much like you, like you, somewhat like you, a little like you, not like you, or not at all like you?

Respondents were then asked to rate a number of statements, including the following statement about adventure, excitement, and risk taking: "Adventure and taking risks are important to this person; to have an exciting life." Respondents rated the statements using a 6-point scale $(1=$ very much like me, $6=$ not at all like me).

In our analyses, we reversed the scale of the item such that the highest value (6) represented the highest propensity for risk taking and the lowest value (1) represented the lowest propensity for risk taking. We also considered a number of demographic variables from the survey, including age and gender, education, marital status, parental status, and current occupational status. These variables represent (a) important indicators of human capital and (b) life-cycle phases that have been hypothesized to influence risk taking (Dohmen et al., 2011; Wilson \& Daly, 1985).

Hardship index. To capture exposure to hardship in each country, we considered a number of indicators that could plausibly capture adversity and economic and social strife: homicide rate, gross domestic product, income inequality, infant mortality, life expectancy at birth, and gender equality (as indexed by the ratio of males to females receiving primary education). We manually compiled data concerning these indicators from the World Health Organization (homicide rate; World Health Organization, 2015), the World Bank (gender equality; World Bank, 2015), and the U.S. Central Intelligence Agency (gross domestic product, income inequality, infant mortality, life expectancy at birth; U.S. Central Intelligence Agency, 2015). By and large, the single indicators were significantly correlated. To obtain a single index representing exposure to hardship in each country, we $z$-standardized all of the indicators and used appropriate transformations (i.e., log transform); some of the indicators required reverse coding. Each indicator had missing data; the number of countries with missing data 
ranged from one ( $1 \%$ of the sample) to nine ( $12 \%$ of the sample). We imputed the missing values with the median of each indicator so that we could use all countries and indicators in our analyses. Overall, the standardized and transformed hardship indicators were reasonably consistent, Cronbach's $\alpha=.86$. Consequently, we obtained a single hardship index by averaging all six $z$-standardized and transformed indicators. The specific data sources, as well as our procedure and the intercorrelations among indicators, are described in detail in the Supplemental Tables and Figures in the Supplemental Material available online.

\section{Statistical analysis}

We opted to use a linear regression approach to model the dependent variable, propensity for risk taking. First, however, we normalized it to have a mean of 50 and a standard deviation of 10 (i.e., $T$ score), which is a common approach when using single, ordinal variables in linear regression models (Stevenson \& Wolfers, 2008). Note, however, that the results were identical for the linear regression and the ordinal logistic regression, but the latter are less straightforward to depict and interpret (see Supplemental Tables and Figures).

We estimated the effects of different independent variables on the risk measure using mixed-effects linear regression in $\mathrm{R}$ ( $\mathrm{R}$ Development Core Team, 2013). Specifically, we used the function lmer in the lme4 package (Bates, Maechler, Bolker, \& Walker, 2014), and we obtained $p$ values for each effect on the basis of Satterthwaite's approximation using the package lmerTest (Kuznetsova, Brockhoff, \& Christensen, 2015). Note that our modeling approach was particularly suited to our research question because it considered fixed effects (i.e., across country, average) of age and gender but also random effects (i.e., country specific) of these factors. This approach permitted us to assess the effect of ecological (i.e., country) characteristics on age and gender differences in propensity for risk taking and to control for other potentially relevant demographic covariates, such as marital status and education.

We report models estimating linear effects of age and gender but no age-by-gender interactions because models including covariates as well as quadratic effects of age or interactions with gender (or both) failed to converge. This finding suggests that the models that included interactions do not provide an appropriate description of the data. In all our analyses, we used age as a continuous variable and binary or dichotomized predictors to simplify coefficient estimation and interpretation, leading to the following additional predictors: gender $(0=$ female, $1=$ male $)$, marital status $(0=$ not married, $1=$ married $)$, parental status $(0=$ no children, $1=$ children $)$, education
$(0=$ no or incomplete primary education, $1=$ primary education or higher), and occupational status $(0=$ not currently employed, 1 = currently employed). We compared different regression models using log-likelihood tests.

\section{Results}

We conducted a number of mixed-effects regression models with propensity for risk taking as the dependent variable. Table 1 presents the fixed-effects coefficients of all relevant models. We first compared a baseline model that did not consider any predictors (intercept-only model; not shown) with Model 1, which included age and gender as predictors. The significantly better fit of Model 1 relative to baseline suggests that age and gender are important predictors that contribute to explaining a

Table 1. Estimated Fixed-Effects Coefficients From the MixedEffects Regression Models of Propensity for Risk Taking Across the 77 Countries

\begin{tabular}{|c|c|c|c|c|}
\hline Model and predictor & $b$ & $S E$ & $\begin{array}{c}T \\
\text { score }\end{array}$ & $\begin{array}{c}p \\
\text { value }\end{array}$ \\
\hline \multicolumn{5}{|l|}{ Model 1: Age + gender } \\
\hline Intercept & 51.46 & 0.29 & 174.99 & $<.001$ \\
\hline Age & -1.98 & 0.11 & -18.15 & $<.001$ \\
\hline Gender & -2.33 & 0.12 & -20.10 & $<.001$ \\
\hline \multicolumn{5}{|c|}{$\begin{array}{l}\text { Model 2: Age }+ \text { gender }+ \\
\text { demographic covariates }\end{array}$} \\
\hline Intercept & 52.09 & 0.27 & 192.40 & $<.001$ \\
\hline Age & -1.43 & 0.09 & -16.19 & $<.001$ \\
\hline Gender & -2.17 & 0.12 & -17.82 & $<.001$ \\
\hline Parental status & -1.32 & 0.13 & -10.27 & $<.001$ \\
\hline Marital status & -0.85 & 0.10 & -8.59 & $<.001$ \\
\hline Occupational status & 0.16 & 0.08 & 2.06 & .04 \\
\hline Education & 0.79 & 0.12 & 6.66 & $<.001$ \\
\hline \multicolumn{5}{|c|}{$\begin{array}{l}\text { Model 3: Age }+ \text { gender }+ \\
\text { demographic covariates }+ \\
\text { hardship }\end{array}$} \\
\hline Intercept & 52.10 & 0.26 & 198.17 & $<.001$ \\
\hline Age & -1.42 & 0.07 & -19.22 & $<.001$ \\
\hline Gender & -2.16 & 0.11 & -18.83 & $<.001$ \\
\hline Parental status & -1.32 & 0.13 & -10.29 & $<.001$ \\
\hline Marital status & -0.85 & 0.10 & -8.70 & $<.001$ \\
\hline Occupational status & 0.16 & 0.08 & 2.02 & .05 \\
\hline Education & 0.79 & 0.12 & 6.83 & $<.001$ \\
\hline Hardship & 0.67 & 0.33 & 2.04 & .04 \\
\hline Hardship $\times$ Age & 0.48 & 0.10 & 4.97 & $<.001$ \\
\hline Hardship $\times$ Gender & 0.29 & 0.14 & 2.03 & .05 \\
\hline
\end{tabular}

Note: Variables were coded as follows-gender: $0=$ male, $1=$ female; parental status: $0=$ no children, $1=$ children; marital status: $0=$ unmarried, $1=$ married; occupational status: $0=$ unemployed, $1=$ employed; and education: $0=$ no or incomplete primary education, $1=$ primary education or higher. 
significant amount of variance in propensity for risk taking, $\chi^{2}(7, N=147,118)=9,293, p<.001$. Table 1 shows that propensity for risk taking tended to decrease as a function of age and was lower for females than for males. We also ran Model 2, which included additional covariates of interest (i.e., education, parental status, marital status, and occupational status). Model 2 provided a significant improvement in fit relative to Model $1, \chi^{2}(26, N=$ $147,118)=1,524, p<.001$, but the results in Table 2 show that the main effects of age and gender remained after the inclusion of the additional demographic predictors.

But to what extent are life-span reductions in propensity for risk taking universal? Figure 2 plots the aggregate results as well as the country-specific effects of age and gender as estimated from Model 2. The pattern of reduction in propensity for risk taking across the life span, as well as the increased propensity for risk taking of males relative to females, was replicated in the vast majority of countries.

Despite the commonalities across countries, Figure 2 also highlights considerable variance in propensity for risk taking; it steeply declined with age in most countries but there are exceptions, such as Nigeria or Mali. As expected from the predictions of life-history theory, variation between countries in propensity for risk taking was associated with local characteristics as captured by our hardship index. Model 3, which included the hardship index as a covariate as well as interactions of hardship with age and gender, provided an additional improvement in fit relative to Model $2, \chi^{2}(3, N=147,118)=$ $124,283, p<.001$. Moreover, the results suggest that hardship was related to intercept differences in propensity for risk taking, as well as the age and gender effects identified in the previous models.

The substantive interpretation of the hardship effects is better captured in Figure 3, which depicts the relation between the random coefficients for each country (i.e., intercepts, age, gender) from the model without hardship as a predictor (Model 2). Figure 3 presents the zero-order correlations between the model coefficients for each country and the hardship index. The hardship index was significantly correlated to the intercept of propensity for risk taking in each country, $r=.24, p<.03$, as well as with the age coefficients, $r=.56, p<.001$, and gender coefficients, $r=.40, p<.001$. For example, Figure 3 shows that the higher the hardship experienced in each country, the closer the age coefficient was to 0 , which represents a flat propensity-for-risk-taking curve across the adult life span.

In summary, we found that harsher environments were associated with increased propensity for risk taking in young adults, smaller gender differences, and smaller differences in propensity for risk taking between younger and older individuals (i.e., a flattening of the age-risk curve). Overall, this finding suggests that ecologically dire circumstances may reduce differences in propensity for risk taking between younger and older individuals.

\section{Discussion}

We analyzed data from a large-scale survey of 77 countries to test whether the typical age-risk progression, which peaks in young adulthood and declines with increased age, represents a pancultural regularity. We found that the overwhelming majority of countries show the typical age-risk pattern, but there is significant variation in the relation between age and propensity for risk taking. Crucially, we found that an index of hardship in each country is significantly associated with the shape of the age-risk function: Hardship is associated with flatter age-risk curves and thus with smaller differences between younger and older age groups and between males and females. In other words, ecologies with scarce resources and therefore heightened competition may lead to increased propensity for risk taking regardless of age and gender. Our work matches expectations from life-history theory that associate ecological characteristics with lifespan development of traits and reproductive strategies (Ellis et al., 2012; Mishra, 2014; Wilson \& Daly, 1985).

Our work has connections to the broader debate about universals in life-span personality development; exploration and risk taking may represent important facets of such development. Whereas some researchers emphasize universals (McCrae et al., 2000), others emphasize the importance of normative as well as idiosyncratic life events in shaping personality; these events are likely to vary across cultures and individuals (Roberts, Wood, \& Smith, 2005). Previous research indicates that there are indeed reliable age differences in personality development, such as decreases in openness to new experiences or increases in conscientiousness with increasing age, that have been replicated across samples and cultures (Roberts, Walton, \& Viechtbauer, 2006). Nevertheless, considerable variation in personality development across cultures is explained by differences in timing of normative life events (Bleidorn et al., 2013). For example, Bleidorn et al. showed that cultures with an earlier onset of adult-role responsibilities, such as starting employment and parenting, were marked by relatively early personality maturation. Our results, which demonstrate a default progression of propensity for risk taking modified by ecological circumstances, are in line with the findings of Bleidorn et al. and with associated theories positing that personality development is a product, at least in part, of experience with the characteristics of local ecologies. Viewed more generally, our work contributes to understanding the causes underlying cultural variation (Weber \& Hsee, 1999) and resonates with calls 


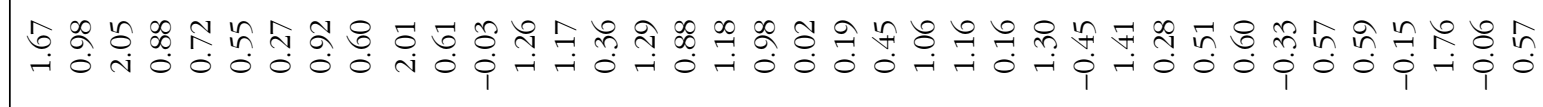

ำ

葛

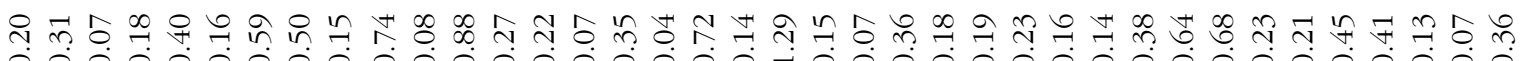

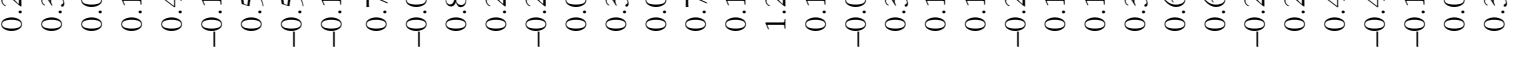

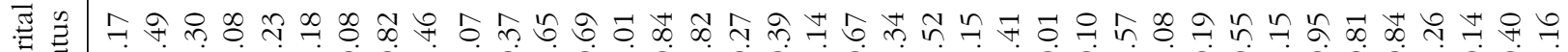
(1)

ił

च्ञ⿹勹巳 氙忽

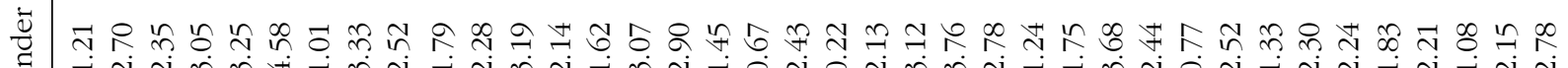
Uू⿹

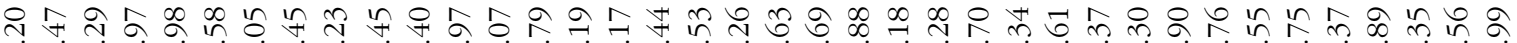

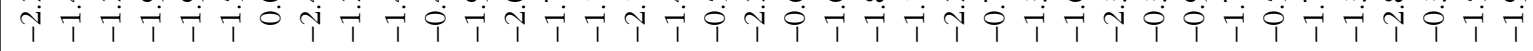

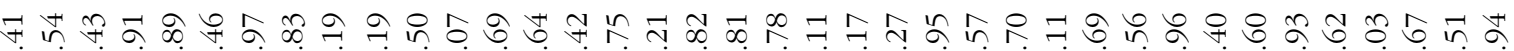

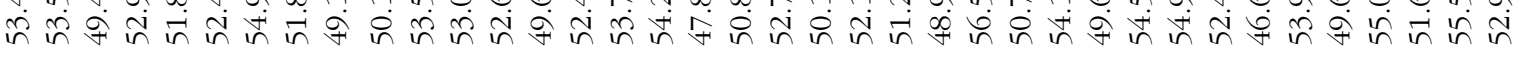

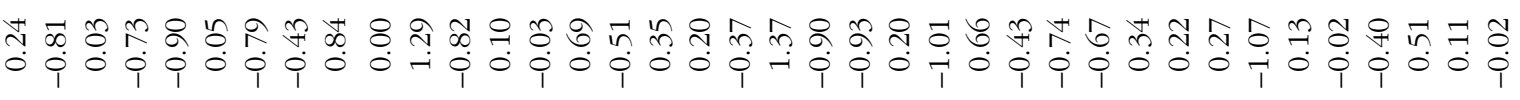

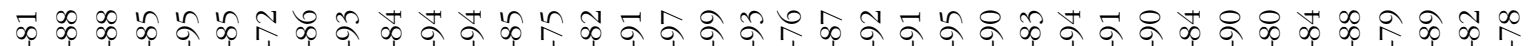

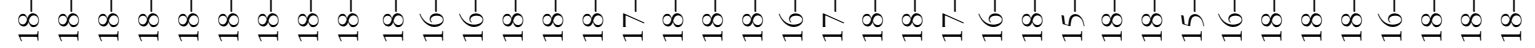

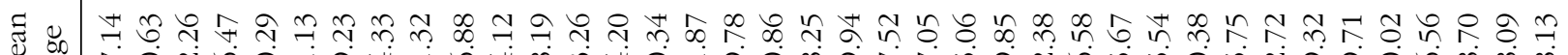

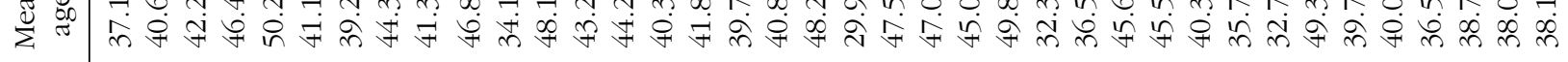

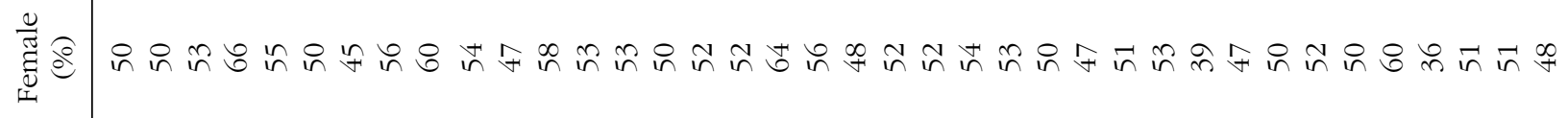

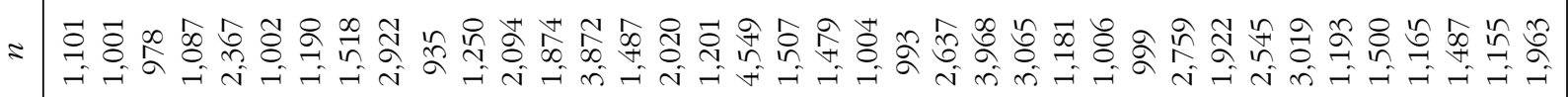

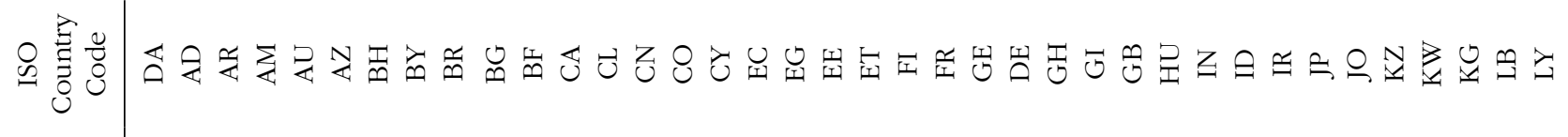

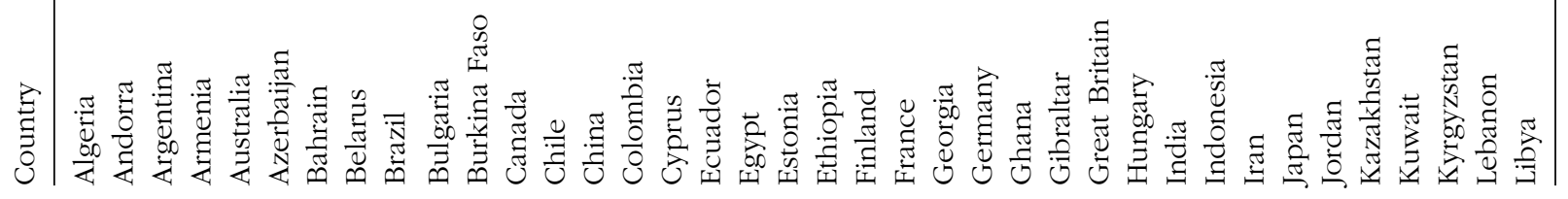




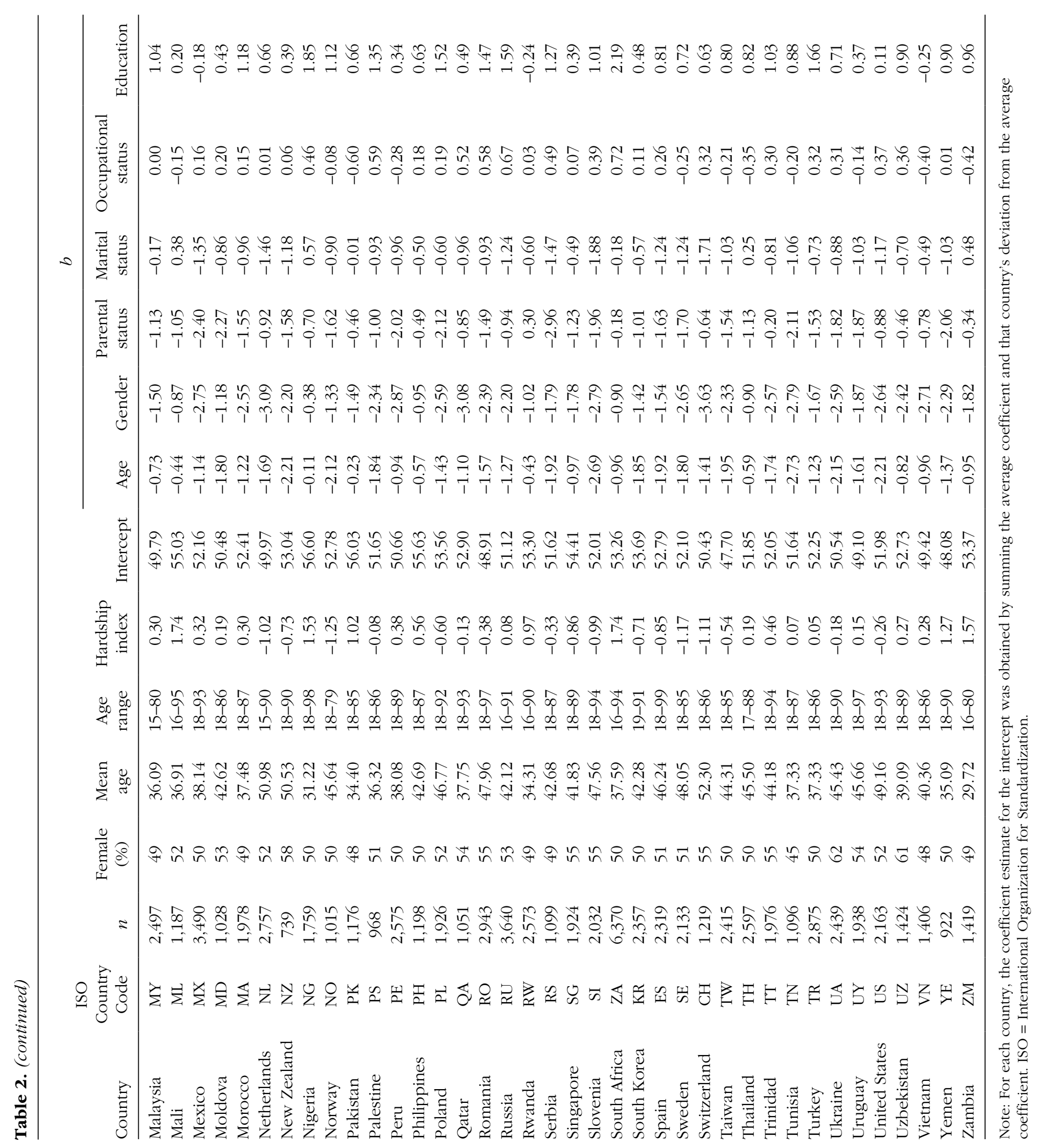




$$
\text { (2) }
$$

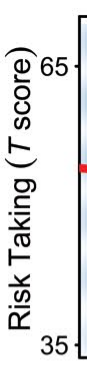
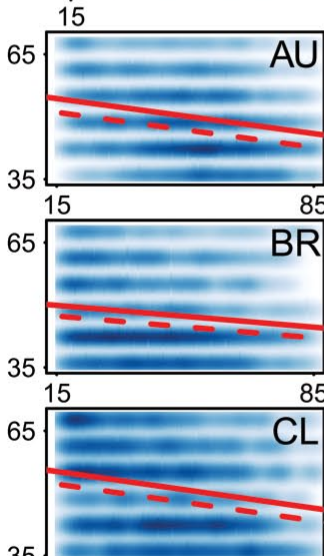

15

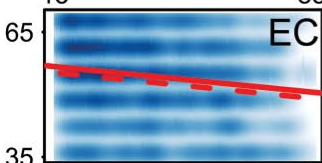

35
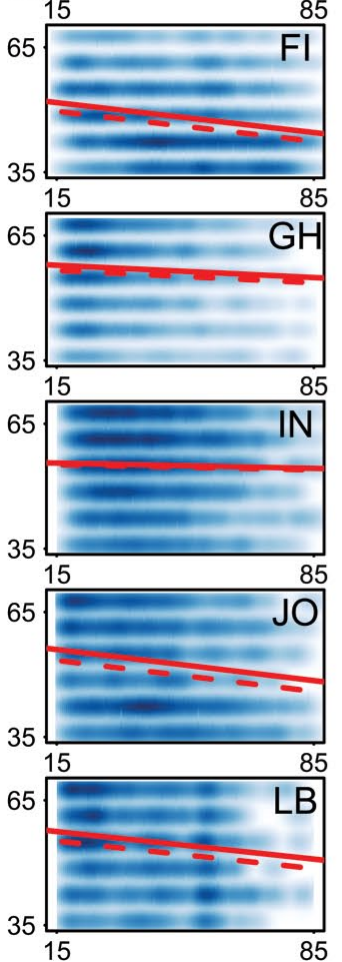

Age

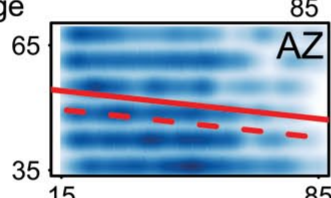

15

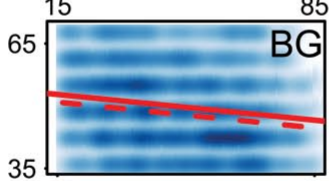

$35 \stackrel{5}{15}$
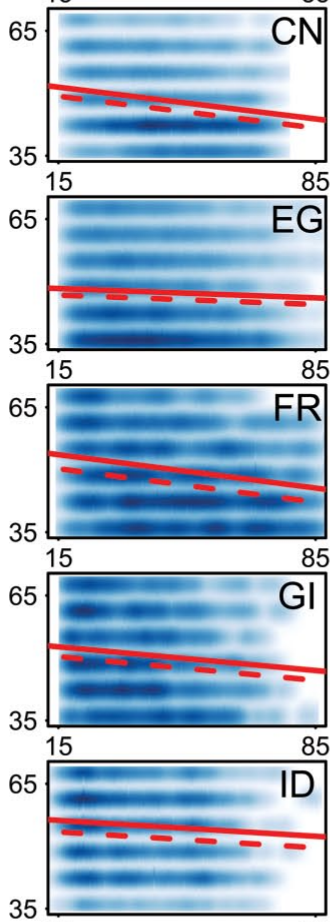

$35 \stackrel{\square}{15}$
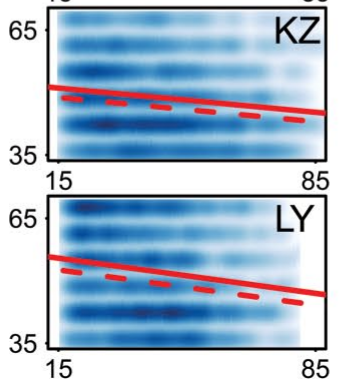

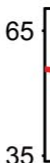

15

65
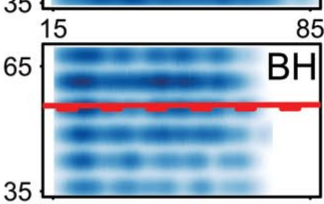

15

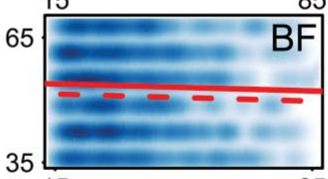

15

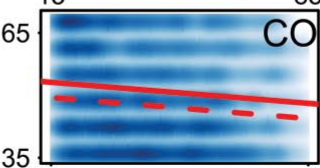

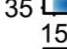

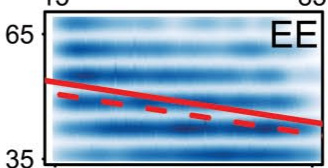

35
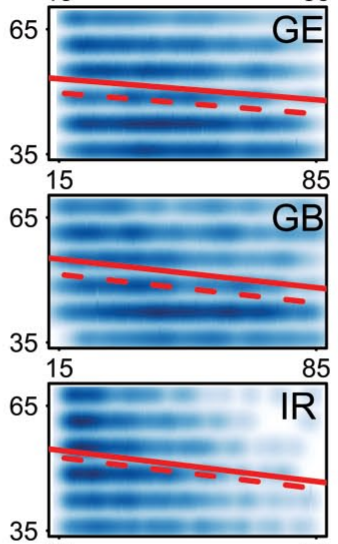

15

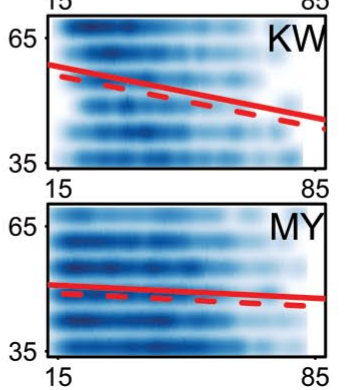

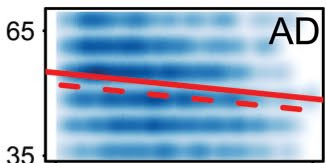
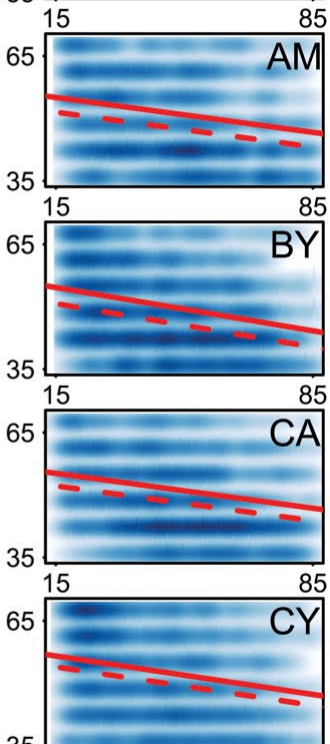

15

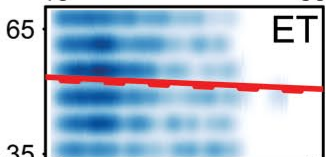

15
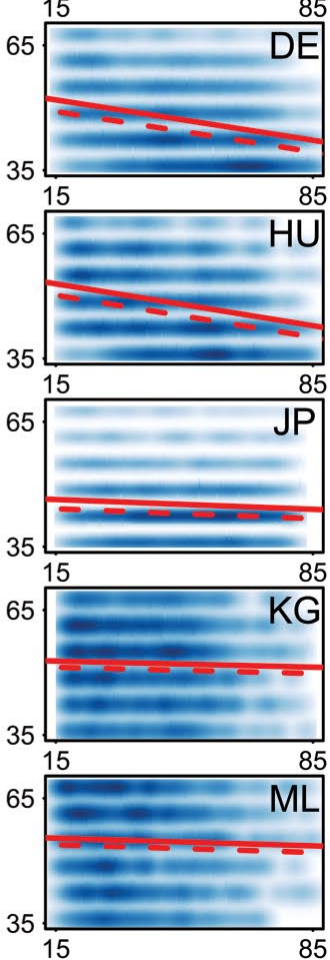

Fig. 2. (continued on next page) 

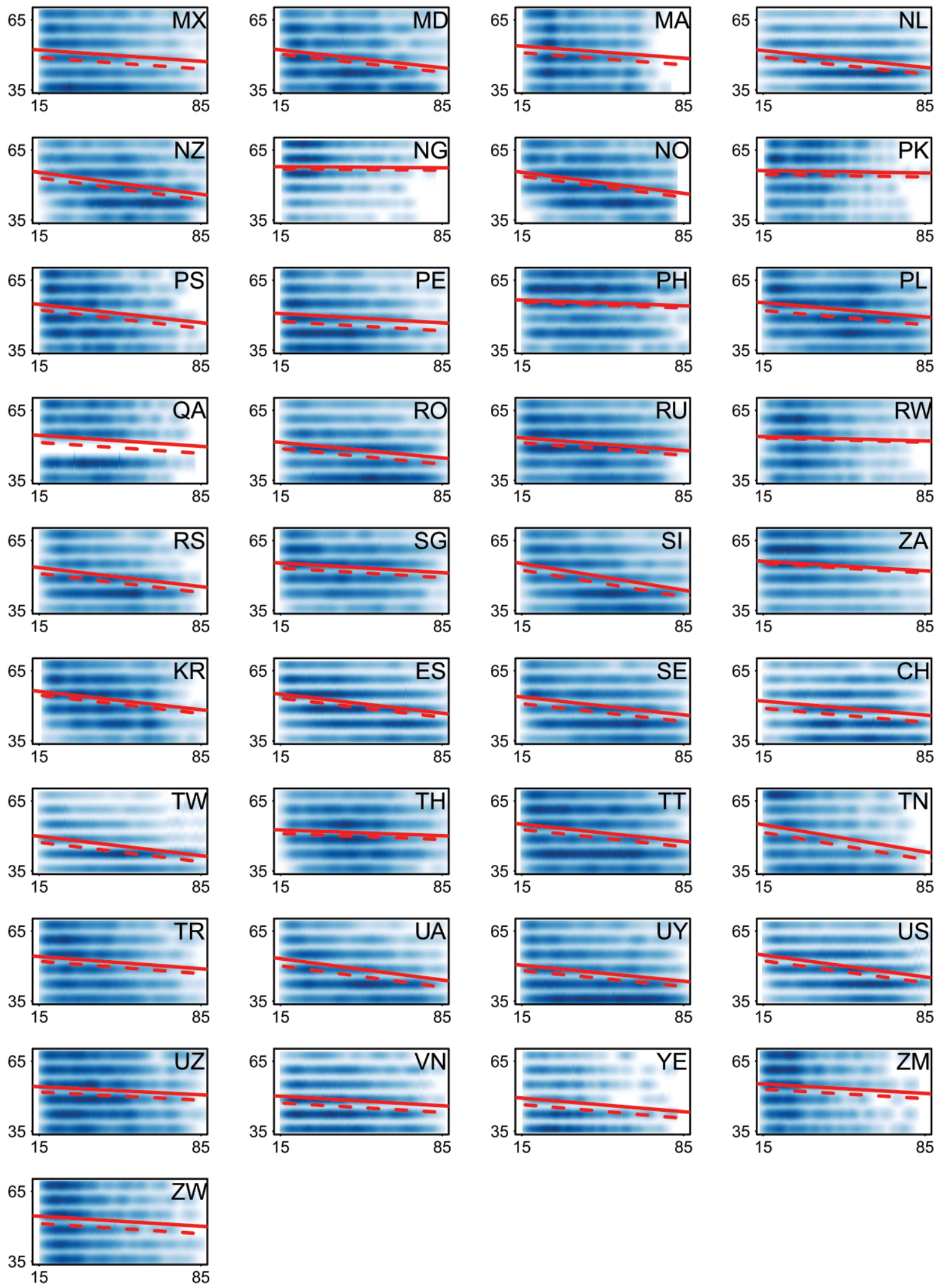

Fig. 2. Density plots of propensity for risk taking as a function of age for all countries combined and for each country separately. The blue background represents the response density; darker colors represent higher densities. Solid lines and dashed lines represent the estimated patterns of propensity for risk taking among males and females, respectively. 
a

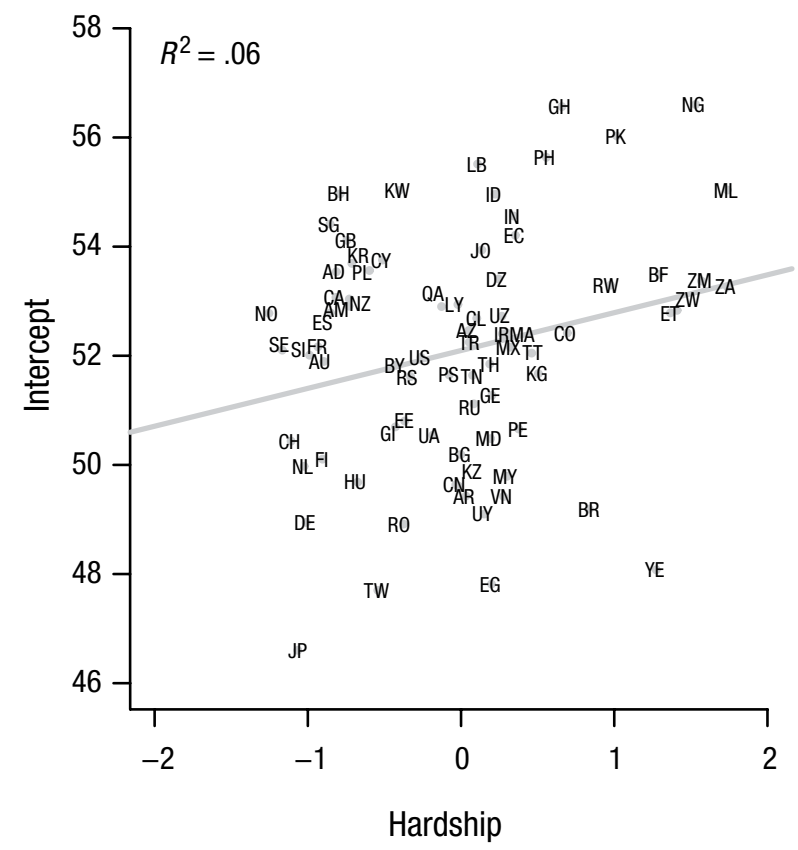

b

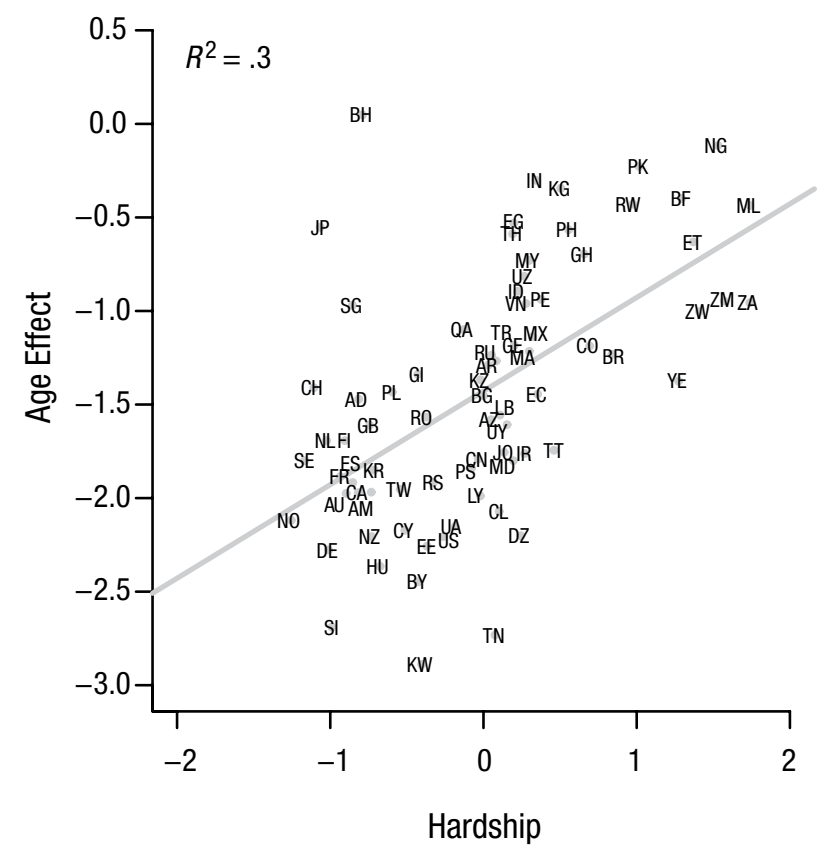

\section{C}

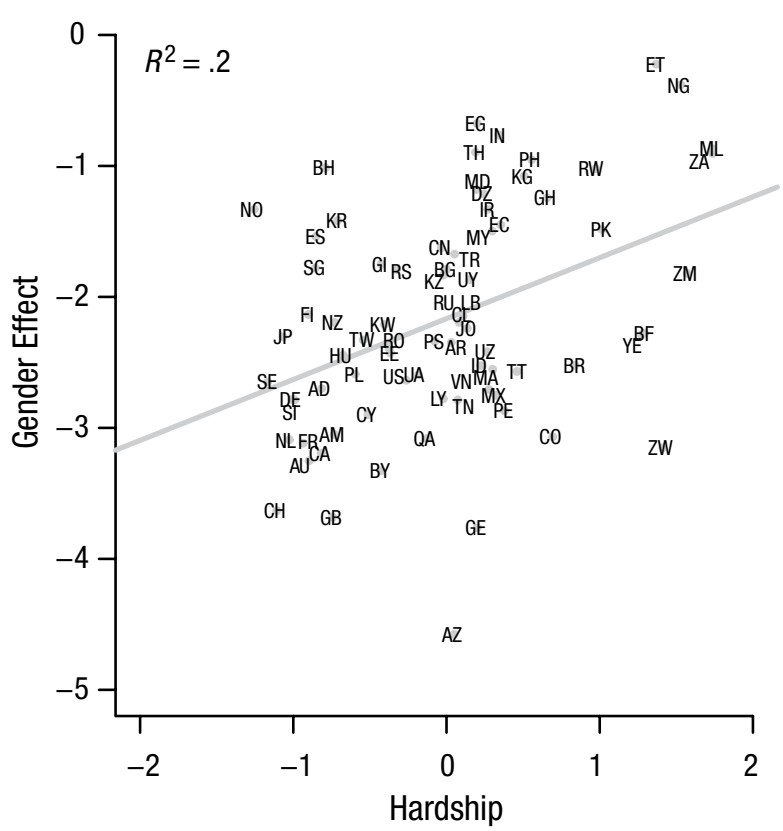

Fig. 3. Scatterplots (with best-fitting regression lines) of the relations between the hardship index and the country-specific (a) intercepts, (b) age-effect estimates, and (c) gender-effect estimates obtained from the mixed-effects regression model in which age and gender (but not hardship) were used to predict risk taking (Model 2). Values on the $y$-axes in (b) and (c) represent deviations from the mean estimate of the effects of age and gender, respectively. See Table 2 for explanations of the country codes.

for the use of diverse samples and cohorts (Henrich, Heine, \& Norenzayan, 2010).

There are a number of limitations associated with the data we report. First, the item "adventure and taking risks are important to this person; to have an exciting life" likely reflects a number of constructs, including propensity for risk taking, sensation seeking, and impulsivity, which are related but not necessarily identical (Zuckerman, 2007). Research on such constructs suggests that each involves distinct components that may merit 
individual investigation (Cross et al., 2013; Mata, Josef, Samanez-Larkin, \& Hertwig, 2011; Sharma, Markon, \& Clark, 2014). Future work may consider other, more specific measures than these that disentangle potential subcomponents of these traits to examine any differential life-span courses between them.

Second, we relied solely on a self-report measure, which may capture current as well as retrospective reports of risk taking and thus cannot assess whether similar patterns would be observed for behavioral measures of risk taking. Past work suggests that there is a correlation, albeit small, between self-reported propensity for risk taking and behavior in economic tasks (Dohmen et al., 2011; Lauriola, Panno, Levin, \& Lejuez, 2013; Mishra \& Lalumière, 2011). However, the pattern of age differences in behavioral measures of risk is considerably heterogeneous; only a few tasks suggest a decline in propensity for risk taking with increased age (Mata et al., 2011). In future work, researchers will need to systematically assess the link between self-report and behavioral measures of propensity for risk taking and do so across cultures (Rieger \& Mata, 2013). Finally, a third limitation of the data is that the meaning of adventure, risk, and excitement is likely to differ between cultures, rendering direct comparisons between countries challenging. Future research may want to relate cultural differences in the perceptions of risk behavior to age differences in propensity for risk taking.

Our work also raises some questions. The monotonic age-related decline in risk taking that we found may not immediately follow from a life-history framework: If risk taking reflects the expected future trajectory of fitness prospects, life-history theory leads to the prediction that very old individuals with increased risk of morbidity and mortality could be more willing to take risks in the hope of immediate successful reproductive efforts, which would lead to a peak in propensity for risk taking in old age (Daly \& Wilson, 2005). Some researchers have suggested that the monotonic reduction in risk taking across the adult life span could be explained by the possibility of resource transfers from older adults (i.e., individuals with lower reproductive value) to their offspring with higher reproductive value (Rogers, 1994). Future work that tracks the risk patterns of older individuals in combination with resource transfer behavior and goals could perhaps test this possibility.

In conclusion, age is associated with reduced propensity for risk taking in a quasi-universal fashion. Nevertheless, the considerable variation in the link between age and propensity for risk taking is systematically associated with local hardship. Specifically, highrisk ecologies favor reproductive strategies associated with increased risk taking across the life span and a flattening of the age-risk curve. Age-risk relations appear to reflect, among other factors, individuals' adjustment to the characteristics of local ecologies and cannot be understood without reference to the demands and affordances of the environment.

\section{Author Contributions}

R. Mata developed the study concept, conducted the analyses, and drafted the manuscript. A. K. Josef and R. Hertwig provided critical revisions. All authors approved the final version of the manuscript for submission.

\section{Declaration of Conflicting Interests}

The authors declared that they had no conflicts of interest with respect to their authorship or the publication of this article.

\section{Funding}

This work was supported by Swiss National Science Foundation Grant 100014-156172.

\section{Supplemental Material}

Additional supporting information can be found at http://pss .sagepub.com/content/by/supplemental-data

\section{Open Practices}

All materials have been made publicly available via Open Science Framework and can be accessed at https://osf.io/ uda75. The complete Open Practices Disclosure for this article can be found at http://pss.sagepub.com/content/by/ supplemental-data. This article has received the badge for Open Materials. More information about the Open Practices badges can be found at https://osf.io/tvyxz/wiki/1.\%20View\%20the\%20 Badges/ and http://pss.sagepub.com/content/25/1/3.full.

\section{References}

Anokhin, A. P., Golosheykin, S., Grant, J., \& Heath, A. C. (2012). Heritability of risk-taking in adolescence: A longitudinal twin study. Twin Research and Human Genetics, 12, 366-371. doi:10.1375/twin.12.4.366

Bates, D., Maechler, M., Bolker, B., \& Walker, S. (2014). lme4: Linear mixed-effects models using Eigen and S4 (Version 1.1-7) [Software]. Retrieved from http://cran.r-project.org/ package $=$ lme 4

Becker, A., Dohmen, T., Enke, B., \& Falk, A. (2014). The ancient origins of the cross-country heterogeneity in risk preferences. Retrieved from https://site.stanford.edu/sites/ default/files/site_enke.pdf

Belsky, J., Schlomer, G. L., \& Ellis, B. J. (2012). Beyond cumulative risk: Distinguishing harshness and unpredictability as determinants of parenting and early life history strategy. Developmental Psychology, 48, 662-673. doi:10.1037/ a0024454

Benjamin, D. J., Cesarini, D., van der Loos, M. J. H. M., Dawes, C. T., Koellinger, P. D., Magnusson, P. K. E., . . . Visscher, 
P. M. (2012). The genetic architecture of economic and political preferences. Proceedings of the National Academy of Sciences, USA, 109, 8026-8031. doi:10.1073/pnas.1120666109

Bezdjian, S., Baker, L. A., \& Tuvblad, C. (2011). Genetic and environmental influences on impulsivity: A meta-analysis of twin, family and adoption studies. Clinical Psychology Review, 31, 1209-1223. doi:10.1016/j.cpr.2011.07.005

Bleidorn, W., Klimstra, T. A., Denissen, J. J. A., Rentfrow, P. J., Potter, J., \& Gosling, S. D. (2013). Personality maturation around the world: A cross-cultural examination of socialinvestment theory. Psychological Science, 24, 2530-2540. doi: $10.1177 / 0956797613498396$

Cross, C. P., Copping, L. T., \& Campbell, A. (2011). Sex differences in impulsivity: A meta-analysis. Psychological Bulletin, 137, 97-130. doi:10.1037/a0021591

Cross, C. P., Cyrenne, D.-L. M., \& Brown, G. R. (2013). Sex differences in sensation-seeking: A meta-analysis. Scientific Reports, 3, Article 2486. doi:10.1038/srep02486

Daly, M., \& Wilson, M. (2005). Carpe diem: Adaptation and devaluing the future. The Quarterly Review of Biology, 80, 55-60. doi:10.1086/431025

Dohmen, T., Huffman, D., Schupp, J., Falk, A., Sunde, U., \& Wagner, G. G. (2011). Individual risk attitudes: Measurement, determinants, and behavioral consequences. Journal of the European Economic Association, 9, 522-550. doi:10.1111/j.1542-4774.2011.01015.x

Ellis, B. J., Del Giudice, M., Dishion, T. J., Figueredo, A. J., Gray, P., Griskevicius, V., . . W Wilson, D. S. (2012). The evolutionary basis of risky adolescent behavior: Implications for science, policy, and practice. Developmental Psychology, 48, 598-623. doi:10.1037/a0026220

Frankenhuis, W. E., \& de Weerth, C. (2013). Does earlylife exposure to stress shape or impair cognition? Current Directions in Psychological Science, 22, 407-412. doi:10.1177/0963721413484324

Henrich, J., Heine, S. J., \& Norenzayan, A. (2010). The weirdest people in the world? Behavioral E Brain Sciences, 33, 61-83. doi:10.1017/S0140525X0999152X

Hsee, C. K., \& Weber, E. U. (1999). Cross-national differences in risk preference and lay predictions. Journal of Behavioral Decision Making, 12, 165-179. doi:10.1002/(SICI)10990771(199906)12:2<165::AID-BDM316>3.0.CO;2-N

Kaplan, H. S., \& Gangestad, S. W. (2005). Life history theory and evolutionary psychology. In D. M. Buss (Ed.), The handbook of evolutionary psychology (pp. 68-96). New York, NY: Wiley.

Kuznetsova, A., Brockhoff, P. B., \& Christensen, R. H. B. (2015). lmerTest: Tests in linear mixed effects models (Version 2.025) [Software]. Retrieved from http://cran.r-project.org/ web/packages/lmerTest/

Lauriola, M., Panno, A., Levin, I. P., \& Lejuez, C. W. (2013). Individual differences in risky decision making: A metaanalysis of sensation seeking and impulsivity with the balloon analogue risk task. Journal of Behavioral Decision Making, 27, 20-36. doi:10.1002/bdm.1784

Mandal, B., \& Roe, B. E. (2014). Risk tolerance among National Longitudinal Survey of Youth participants: The effects of age and cognitive skills. Economica, 81, 522-543. doi:10.1111/ ecca. 12088
Mata, R., Josef, A. K., Samanez-Larkin, G. R., \& Hertwig, R. (2011). Age differences in risky choice: A meta-analysis. Annals of the New York Academy of Sciences, 1235, 18-29. doi:10.1111/j.1749-6632.2011.06200.x

McCrae, R. R., Costa, P. T., Ostendorf, F., Angleitner, A., Hrebícková, M., Avia, M. D., . . Smith, P. B. (2000). Nature over nurture: Temperament, personality, and life span development. Journal of Personality and Social Psychology, 78, 173-186. doi:10.1037//0022-3514.7S.1.173

Mishra, S. (2014). Decision-making under risk: Integrating perspectives from biology, economics, and psychology. Personality and Social Psychology Review, 18, 280-307. doi:10.1177/1088868314530517

Mishra, S., \& Lalumière, M. L. (2011). Individual differences in risk-propensity: Associations between personality and behavioral measures of risk. Personality and Individual Differences, 50, 869-873. doi:10.1016/j.paid.2010.11.037

Quetelet, A. (2013). A treatise on man and the development of his faculties (R. Knox, Ed.). New York, NY: Cambridge University Press. (Original work published 1842)

R Development Core Team. (2013). R: A language and environment for statistical computing (Version 3.1.2) [Computer software]. Retrieved from https://www.r-project.org/index .html

Rieger, M., \& Mata, R. (2013). On the generality of age differences in social and nonsocial decision making. The Journals of Gerontology, Series B: Psychological Sciences $\varepsilon$ Social Sciences, 70, 202-214. doi:10.1093/geronb/gbt088

Rieger, M. O., Wang, M., \& Hens, T. (2015). Risk preferences around the world. Management Science, 61, 637-648. doi: $10.1287 / \mathrm{mnsc} .2013 .1869$

Roberts, B. W., Walton, K. E., \& Viechtbauer, W. (2006). Patterns of mean-level change in personality traits across the life course: A meta-analysis of longitudinal studies. Psychological Bulletin, 132, 1-25. doi:10.1037/00332909.132.1.1

Roberts, B. W., Wood, D., \& Smith, J. L. (2005). Evaluating Five Factor Theory and social investment perspectives on personality trait development. Journal of Research in Personality, 39, 166-184. doi:10.1016/j.jrp.2004.08.002

Rogers, A. R. (1994). Evolution of time preference by natural selection. The American Economic Review, 84, 460-481. Retrieved from http://www.jstor.org/stable/2118062

Schwartz, S. H. (2012). An overview of the Schwartz theory of basic values. Online Readings in Psychology and Culture, 2(1), 1-20. doi:10.9707/2307-0919.1116

Sharma, L., Markon, K. E., \& Clark, L. A. (2014). Toward a theory of distinct types of "impulsive" behaviors: A metaanalysis of self-report and behavioral measures. Psychological Bulletin, 140, 374-408. doi:10.1037/a0034418

Sih, A., \& Del Giudice, M. (2012). Linking behavioural syndromes and cognition: A behavioural ecology perspective. Philosophical Transactions of the Royal Society B: Biological Sciences, 367, 2762-2772. doi:10.1098/rstb.2012.0216

Simpson, J. A., Griskevicius, V., Kuo, S. I.-C., Sung, S., \& Collins, W. A. (2012). Evolution, stress, and sensitive periods: The influence of unpredictability in early versus late childhood on sex and risky behavior. Developmental Psychology, 48, 674-686. doi:10.1037/a0027293 
Steinberg, L. (2008). A social neuroscience perspective on adolescent risk-taking. Developmental Review, 28, 78-106. doi:10.1016/j.dr.2007.08.002

Steinberg, L. (2013). The influence of neuroscience on US Supreme Court decisions about adolescents' criminal culpability. Nature Reviews Neuroscience, 14, 513-518. doi:10.1038/nrn3509

Stevenson, B., \& Wolfers, J. (2008). Economic growth and subjective well-being: Reassessing the Easterlin paradox (NBER Working Paper No. 14282). Retrieved from the National Bureau of Economic Research Web site: http://www.nber .org/papers/w14282

Tooby, J., \& Cosmides, L. (1990). The past explains the present: Emotional adaptations and the structure of ancestral environments. Ethology and Sociobiology, 11, 375-424. doi:10.1016/0162-3095(90)90017-Z

Ulmer, J. T., \& Steffensmeier, D. (2014). The age and crime relationship: Social variation, social explanations. In K. Beaver, B. Boutwell, \& J. C. Barnes (Eds.), The nurture versus biosocial debate in criminology: On the origins of criminal behavior and criminality (pp. 377-396). London, England: Sage. doi:10.4135/9781483349114.n24

U.S. Central Intelligence Agency (2015). The world factbook. Retrieved from https://www.cia.gov/library/publications/ the-world-factbook

Vieider, F. M., Chmura, T., Fisher, T., Kusakawa, T., Martinsson, P., Mattison Thompson, F., . . . Sunday, A. (2015). Within- versus between-country differences in risk attitudes: Implications for cultural comparisons. Theory and Decision, 78, 209-218. doi:10.1007/s11238-014-9418-3

Weber, E. U., \& Hsee, C. K. (1999). Models and mosaics: Investigating cross-cultural differences in risk perception and risk preference. Psychonomic Bulletin \& Review, 6, 611-617. doi:10.3758/BF03212969

Wilson, M., \& Daly, M. (1985). Competitiveness, risk taking, and violence: The young male syndrome. Ethology and Sociobiology, 6, 59-73. doi:10.1016/0162-3095(85)90041-X

Wilson, M., \& Daly, M. (1997). Life expectancy, economic inequality, homicide, and reproductive timing in Chicago neighbourhoods. British Medical Journal, 314, 1271-1274. doi:10.1136/bmj.314.7089.1271

World Bank. (2015). World databank. Retrieved from http:// databank.worldbank.org/data/

World Health Organization. (2015). Global health observatory data repository. Retrieved from http://apps.who.int/gho/data

World Values Survey Association. (2008). Wave 5 2005-2008 official aggregate v.20140429. Retrieved from http://www .worldvaluessurvey.org/WVSDocumentationWV5.jsp

World Values Survey Association. (2014). Wave 6 2010-2014 official aggregate v.20140429. Retrieved from http://www .worldvaluessurvey.org/WVSDocumentationWV6.jsp

Zuckerman, M. (2007). Sensation seeking and risky behavior. Washington, DC: American Psychological Association. doi:10 $.1037 / 11555-000$ 